\title{
Combined Myocardial Infarction in a Young Patient with Anomalous Coronary Artery Anatomy: A Case Report
}

\author{
Hadadi László ${ }^{1,2 *}$, Lakatos Éva Katalin ${ }^{1,2}$, Țăruși Mariana², Dobreanu Dan ${ }^{1,2}$ \\ 1 Univeristy of Medicine and Pharmacy of Tîrgu Mureș, Romania \\ 2 Emergency Institute for Cardiovascular Diseases and Transplantation of Tîrgu Mureș, Romania
}

\begin{abstract}
Introduction: combined myocardial infarction (MI) is defined as the simultaneous ischemic injury of two different myocardial territories, raising the possibility of multiple culprit lesions. The anomalous origin of a coronary artery could represent an important challenge during percutaneous coronary intervention (PCl). Case presentation: A 46 year old, smoker Caucasian male presented to a territorial hospital four hours after the onset of severe angina. Consecutive electrocardiograms showed complete atrioventricular block and inferior ST segment elevation (STE), later PQ prolongation with right bundle branch block plus STE in leads V4-V6. After administration of thrombolytic treatment, the patient was transferred to the regional $\mathrm{PCl}$ center. Emergent coronary angiography revealed acute occlusion of the left anterior descending artery (LAD) and a thrombus containing, severe stenosis of the anomalously originated right coronary artery (RCA). Rescue PCl with stent implantation in the LAD and RCA was performed nine hours after pain onset. At the 1 year follow-up visit the patient had no angina or heart failure symptoms. Conclusion: this is the first report of a combined Ml caused by acute, sequentially occurring thrombotic occlusion of two coronary arteries, one of them with anomalous origin, in a patient treated by rescue PCl following partially successful thrombolysis.
\end{abstract}

Keywords: combined myocardial infarction, coronary artery anomaly, thrombolysis, rescue percutaneous coronary artery intervention

Received: 18 August 2015 / Accepted: 11 September 2015

\section{Introduction}

The term of double or combined myocardial infarction (MI) is defined as the simultaneous ischemic injury of two different myocardial territories, raising the possibility of multiple culprit lesions [1]. This condition is rarely reported in clinical studies [2], but there is intravascular ultrasound evidence that multiple unstable plaques coexist in different major epicardial coronary artery branches at the time of an acute coronary syndrome [3]. The simultaneous occlusion of two major coronary arteries is frequently associated with hemodynamic and electrical instability and requires a time-limited and challenging management [4]. Although the preferred treatment of the acute MI with ST segment elevation (STEMI) is primary percutaneous coronary intervention (PCI) $[5,6]$, the anomalous origin of a coronary artery could represent an important challenge during this procedure, contributing to additional delay before revascularization $[7,8]$.

Case presentation: A 46 year old Caucasian male, with no comorbidities or cardiovascular risk factors except smoking, presented to a territorial hospital four hours after the onset of severe typical chest pain. Physical examination revealed: blood pressure 100/60 $\mathrm{mmHg}$, heart rate: 45 beats/min and bibasilar lung rales. The first electrocardiogram (ECG) showed sinus rhythm with complete atrioventricular block and inferior STE (figure 1).

* Correspondence to: László Hadadi

E-mail: hadadilaci@yahoo.com
On repeated ECG appeared only PQ prolongation, but with right bundle branch block (RBBB) and $1 \mathrm{~mm}$ STE in leads V4-V6 besides the inferior STE. After administration of full dose thrombolytic treatment, the patient was immediately transferred to the regional PCI center, the Emergency Institute for Cardiovascular Diseases and Transplantation of Tîrgu Mureș. He arrived with persistent angina, in Killip class II, with STE resolution $>50 \%$ in the inferior leads, but persistence of $\mathrm{PQ}$ prolongation, RBBB and $2.5 \mathrm{~mm} \mathrm{STE}$ in leads V2-V6 (figure 2).

Emergent coronary angiography (figure 3) revealed acute occlusion of the middle left anterior descending artery and a thrombus containing, severely stenotic lesion in the second segment of the anomalously originated right coronary artery (RCA). No external compression of the

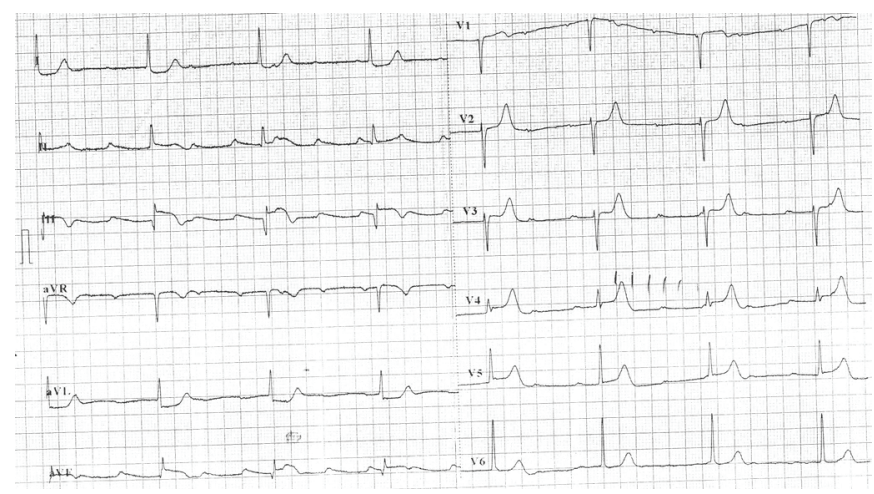

Fig. 1. Electrocardiogram at first medical contact: ST-segment elevation in the inferior leads, associated with complete atrioventricular block. 


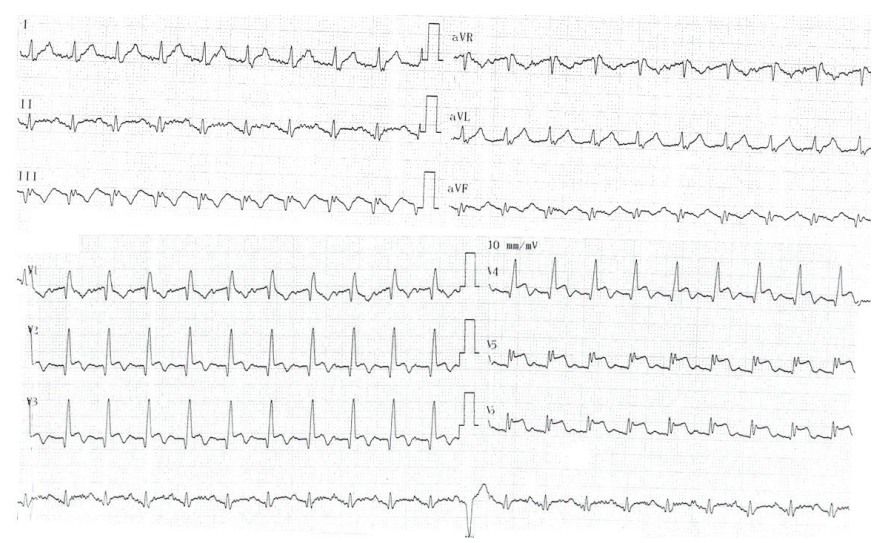

Fig. 2. Electrocardiogram recorded at the $\mathrm{PCl}$ centre, immediately before coronary angiography. A newly appeared right bundle branch block is associated with antero-lateral ST-segment elevation.

proximal RCA was noted. The selective cannulation of the RCA was possible with Judkins Left catheters only. Rescue PCI with implantation of 2 bare metal stents in the $\mathrm{LAD}$ and RCA, respectively, was performed nine hours after pain onset, using the same Judkins Left 4.0 guiding catheter (figure 3).

After an uneventful in-hospital evolution, the patient was discharged asymptomatically, with a left ventricular ejection fraction of $35 \%$. At the 6-months follow-up visit he had no angina or heart failure symptoms. At this time, computed tomography angiography revealed patent stents (figure 4) and confirmed the high, acute-angle takeoff of the RCA arising above the sinotubular junction, with the

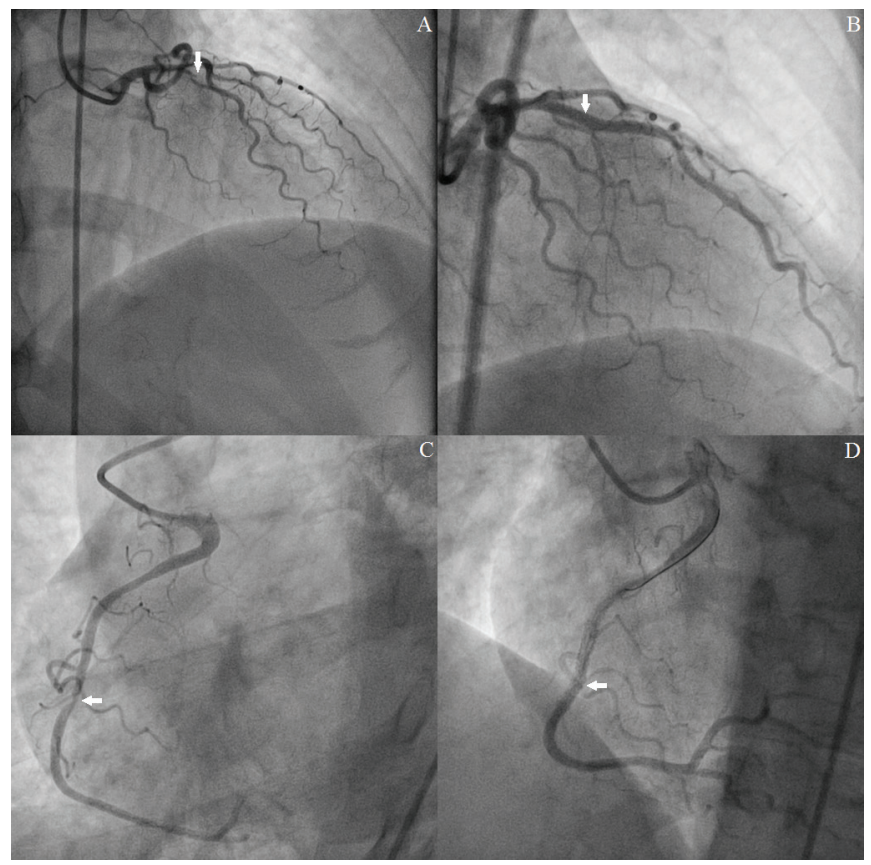

Fig. 3. Coronary angiography before and after rescue percutaneous coronary intervention. A. complete thrombotic occlusion (arrow) of the left anterior descending coronary artery. B. Final result after stent implantation (arrow). C. Severe, thrombus-containing lesion (arrow) in the right coronary artery with anomalous origin. D. After stent implantation (arrow) an excellent angiographic result is obtained. first segment situated between the aorta and the pulmonary artery (figure 4).

At 1 year after de acute event the patient is still doing well, without significant symptoms (figure 5).

\section{Discussions}

Although very rarely reported in clinical registries[2], pathologic evidence of simultaneous thrombotic occlusion of more than one coronary artery is not uncommon[9]. This could be explained by the fact that these patients often have extensive myocardial injury and die before arriving to the hospital [10]. As clinical evidence is mainly based on case reports, it is little known about the pathophysiology of double coronary artery thrombosis $[1,4,10,11]$. It is supposed that the prothrombotic and proinflammatory conditions associated with the first MI may provoke additional acute thrombosis of other unstable atherosclerotic lesions, especially in the case of sequentially appearing coronary artery thrombosis during the same acute clinical event [10].

In our case the ECG proved to be a good and valuable diagnostic tool and an important prognostic indicator, its aspect correlating well with the angiographic findings and thus help to stratify the patient for more aggressive and appropriate forms of therapy. Although thrombolysis was successful only in the case of the RCA occlusion, it was probably life-saving. According to current guidelines, multivessel PCI in acute MI patients with STE is reserved for cases with cardiogenic shock, where all critical lesions should be addressed during the same emergency interventional procedure $[5,6]$. However,in the absence of shock,

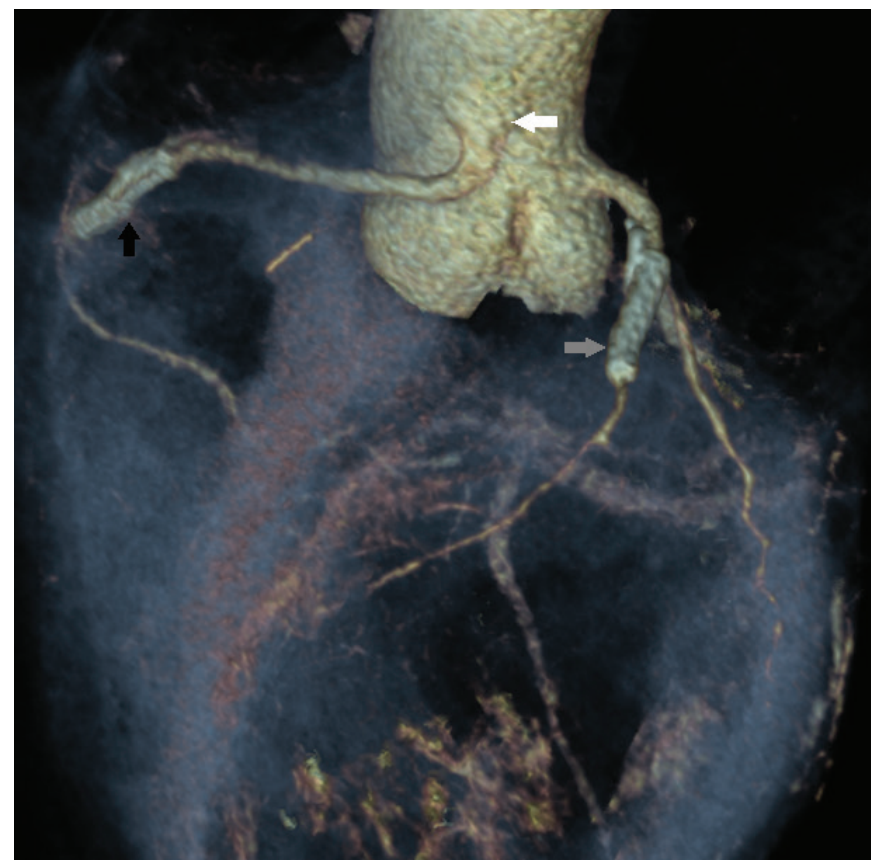

Fig. 4. Three-dimensional reconstruction of coronary computed tomography angiography. The origin of the right coronary artery (white arrow) is situated far above the commissure between the left and right cusps of the aortic valve. The stents implanted in the left anterior descending coronary artery (grey arrow) and in the right coronary artery (black arrow) are also visualized. 


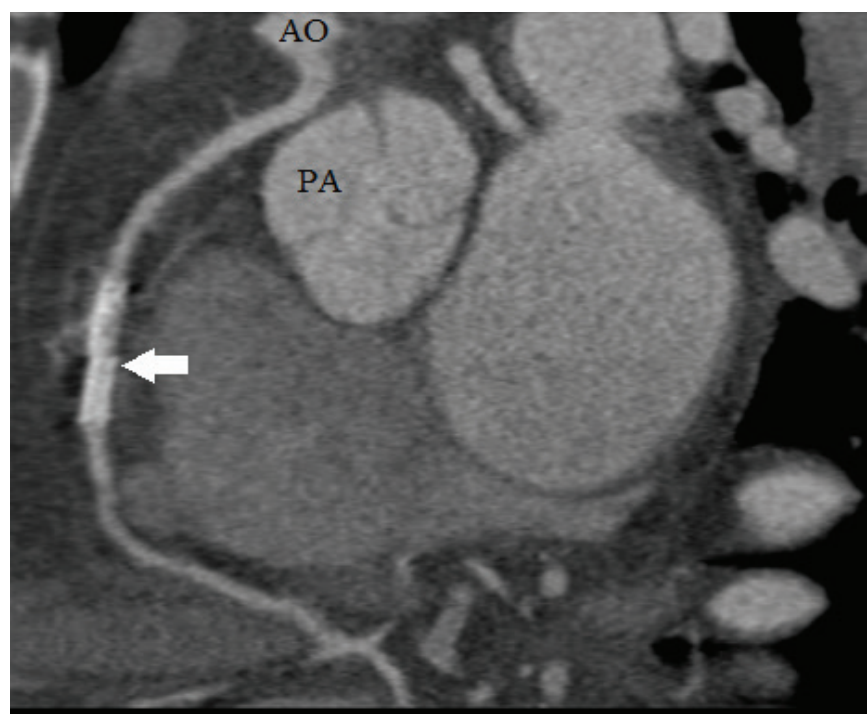

Fig. 5. Coronary computed tomography angiographic image showing the interarterial course of the proximal right coronary artery situated between the aorta (AO) and the pulmonary artery (PA). The stent of the second segment is indicated by arrow.

there are no specific recommendations for cases with multiple culprit lesions. Although there was no flow-limitation in the RCA after thrombolysis, considering the electrocardiographic and angiographic findings, PCI of the RCA was attempted during the same interventional procedure. In addition, due to the anomalous origin of the RCA, the same guiding catheter could be used for the interventional treatment of both acute lesions. Although Judkins left guiding catheters are feasible for primary PCI of culprit lesions localized in RCA with anomalous origin [7], the intervention is done with reduced back-up support [8]. In addition to the increased difficulty of the PCI procedure, the anomalous proximal interarterial course of the RCA between the aorta and the pulmonary artery could have further ischemic consequences [12]. However, given the absence of luminal compression on angiography and the asymptomatic long-term evolution, surgical correction of the congenital anomaly was not proposed. Despite the complexity of the case, the combination of reperfusion therapies resulted in a good immediate outcome, followed by favourable long-term evolution.

\section{Conclusions}

This is the first report of a combined MI caused by acute, sequentially occurring thrombotic occlusion of two coronary arteries, one of them with anomalous origin, in a patient treated by rescue PCI following partially successful thrombolysis. The combined, pharmaco-invasive strategy appears to be a feasible approach for the treatment of these critically ill patients.

\section{Acknowledgement}

This paper was published under the frame of European Social Found, Human Resources Development Operational Programme 2007-2013, project no. POSDRU/159/1.5/S/136893.

\section{Conflict of interest}

The authors declare that they have no conflict of interest.

\section{References}

1. Toutouzas K, Synetos A, Karanasos A, Tsiamis E, Stefanadis C. Simultaneous occlusion of two coronary arteries in a patient with acute myocardial infarction: a result of plaque rupture and plaque erosion. International Journal of Cardiology. 2011;152:e29-30.

2. Pollak PM, Parikh SV, Kizilgul M, Keeley EC. Multiple culprit arteries in patients with ST segment elevation myocardial infarction referred for primary percutaneous coronary intervention. The American Journal of Cardiology. 2009;104:619-623.

3. Rioufol G, Finet G, Ginon I, et al. Multiple atherosclerotic plaque rupture in acute coronary syndrome: a three-vessel intravascular ultrasound study. Circulation. 2002;106:804-808.

4. Sia S-K, Huang C-N, Ueng K-C, Wu Y-L, Chan K-C. Double vessel acute myocardial infarction showing simultaneous total occlusion of left anterior descending artery and right coronary artery. Circulation Journal: Official Journal of the Japanese Circulation Society. 2008;72:1034-1036.

5. Steg PG, James SK, Atar D, et al. ESC Guidelines for the management of acute myocardial infarction in patients presenting with ST-segment elevation. European heart journal. 2012;33:2569-2619.

6. O'Gara PT, Kushner FG, Ascheim DD, et al. 2013 ACCF/AHA Guideline for the Management of ST-Elevation Myocardial Infarction: A Report of the American College of Cardiology Foundation/American Heart Association Task Force on Practice Guidelines. Journal of the American College of Cardiology. 2013;61:e78-e140.

7. Matchison JC, Shavelle DM. Primary percutaneous coronary intervention of an anomalous right coronary artery arising from the left coronary cusp using an undersized Judkins catheter: A case report. The International Journal of Angiology: Official Publication of the International College of Angiology, Inc. 2007;16:33-35.

8. Shuaib W, Arepalli C, Vijayasarathi A, et al. Coronary anomalies encountered in the acute setting: an imaging review. Emergency Radiology. 2014;21:631-641.

9. Davies MJ, Thomas A. Thrombosis and acute coronary-artery lesions in sudden cardiac ischemic death. The New England Journal of Medicine. 1984;310:1137-1140.

10. Ribeiro H, Ferreira C, Batista A, et al. [Sequential double vessel myocardial infarction]. Revista Portuguesa De Cardiologia: Orgão Oficial Da Sociedade Portuguesa De Cardiologia = Portuguese Journal of Cardiology: An Official Journal of the Portuguese Society of Cardiology. 2011;30:925-927.

11. Magno P, Farto e Abreu P, Baptista SB, Gil VM. Combined myocardial infarction: two case reports. Revista Portuguesa De Cardiologia: Orgão Oficial Da Sociedade Portuguesa De Cardiologia = Portuguese Journal of Cardiology: An Official Journal of the Portuguese Society of Cardiology. 2008;27:1157-1165.

12. Nasis A, Machado C, Cameron JD, et al. Anatomic characteristics and outcome of adults with coronary arteries arising from an anomalous location detected with coronary computed tomography angiography. The International Journal of Cardiovascular Imaging. 2015;31:181-191. 